\title{
PREDOMINANT TUBULOINTERSTITIAL INVOLVEMENT IN LUPUS NEPHRITIS: CASE REPORT AND BRIEF REVIEW OF THE LITERATURE
}

Larissa Maria Oliveira Gonzaga'^^, Ana Luisa Bagno de Almeida', Victória Carneiro Dal Moro', Débora Cerqueira Calderaro', Anna Carolina Faria Moreira Gomes Tavares ${ }^{1}$, Maria Raquel da Costa Pinto', Gilda Aparecida Ferreira ${ }^{1}$

1.Universidade Federal de Minas Gerais, Belo Horizonte (MG), Brazil.

*Corresponding author: gonzagamlarissa@gmail.com

\section{BACKGROUND}

Renal involvement is one of the main determinants of morbidity and mortality in systemic lupus erythematosus (SLE). Recently, an increasing body of evidence suggests the importance of tubulointerstitial damage as a predictor of unfavorable outcomes in lupus nephritis (LN). Thus, a greater emphasis on the description of tubulointerstitial compartment in renal biopsies has been suggested, since glomerular and tubulointerstitial lesions do not always coexist and findings of tubulointerstitial inflammation, tubular atrophy and/or interstitial fibrosis were independent risk factors for renal outcomes. In this study, the case of one patient presenting LN with a predominant tubulointerstitial involvement is described.

\section{CASE REPORT}

An 18-year-old female patient, previously healthy, presenting pancytopenia, panserositis, acute renal failure, myocarditis with heart failure, fever, hepatosplenomegaly, lymphadenopathy, FAN + >1/640 (anti-RNP +; anti-SM +) was diagnosed with SLE. Treatment with prednisone $1 \mathrm{mg} / \mathrm{kg} /$ day and immunoglobulin IV (IVIG) was introduced; however, she progressed with worsening renal function and a 24-hour urine protein test of $3.8 \mathrm{~g}$. Renal biopsy was performed and presented findings of LN class IV + V/chronicity 8 out of 12 , with predominance of tubular atrophy and interstitial fibrosis. Treatment with IV cyclophosphamide (CYC) plus methylprednisolone was initiated.

\section{CONCLUSION}

Different tubulointerstitial lesions have been described, isolated or in association with different classes of LN, mostly class IV. The real prevalence of tubulointerstitial nephritis (TIN) in LN is probably underestimated, since renal biopsy is not widely available. Tubulointerstitial nephritis has been described in association or not with the finding of immune deposits along the tubular basement membrane. Tubulointerstitial inflammation, tubular atrophy and/or interstitial fibrosis in SLE are associated with poor prognosis, hypertension and progressive clinical course to renal failure. The mechanisms underlying the development of tubulointerstitial lesions in LN remain unclear, and thus there are no specific treatment options. In the cases of LN presenting with predominant TIN, treatment with high dose steroids has been advocated. In the case presented, severe LN with predominant TIN, associated to other severe SLE manifestations, prompted the initiation of high dose corticosteroids combined with IVIG and, lately, IV CYC, with clinical improvement. The growing recognition of TIN as a prognostic factor in $L N$ warrants other studies for explorations into the pathogenesis of tubulointerstitial lesions and the identification of effective therapeutic interventions. 